\title{
Glückwünsche zum bevorstehenden 50. Geburtstag von Univ.-Prof. Dipl.-Ing. Dr. mont. Helmut Flachberger
}

\author{
Günter Waldl \\ Golling, Österreich
}

Im Namen des Fachausschusses für Aufbereitung übermittle ich herzliche Glückwünsche zum runden Geburtstag.

Persönlich verbindet Herrn Prof. Helmut Flachberger und mich nicht nur die gemeinsame Tätigkeit im Fachausschuss für Aufbereitung, sondern eine langjährige Freundschaft, zurückgehend auf die gemeinsame Studienzeit an der Montanuniversität.

Nach einigen Jahren selbständiger Unternehmertätigkeit hat er sich seit der Übernahme der Leitung des Lehrstuhls für Aufbereitung und Veredlung an der Montanuniversität Leoben intensiv um den Fachausschuss für Aufbereitung gekümmert. Seine organisatorischen Tätigkeiten tragen wesentlich zum Gelingen des jährlich stattfindenden Aufbereitungstechnischen Seminars sowie zur erfolgreichen Herausgabe des "Aufbereiterheftes“ der BHM bei. Nicht unerwähnt darf die gemeinsame Herausgabe der Festschriften "Aufbereitung in Österreich, Teil I - III", bleiben. Ohne den unermüdlichen Einsatz von Prof. Flachberger wäre hier vieles nicht und vor allem nicht in dieser Qualität möglich gewesen.

Sicherlich nicht leicht war der Schritt, das Rittinger-Gebäude, in dem das Institut für Aufbereitung untergebracht war, zu verlassen und neue Räumlichkeiten im IZ-Rohstoffe zu beziehen. Der Fachausschuss war in die Planungen eng mit eingebunden. So konnte die Unterstützung von Fachausschussmitgliedern einen Teil zur Errichtung eines modernen Aufbereitungslabors beitragen.

Prof. Flachberger ist dafür bekannt, dass er neben der Leitung des Lehrstuhls viele übergeordnete Ämter und Tätigkeiten übernimmt, was einen disziplinierten Umgang mit der Ressource Zeit erfordert. Hier unterstützt ihn sicherlich seine Konsequenz, die er auch durch die Ausbildung zum Milizoffizier perfektioniert hat.

Er vergisst (hoffentlich) daneben nicht auf sein Privatleben, auf seine Frau, Dr. Sonja Flachberger, und die drei Kinder Wolfgang, Valerie und Matthias. Gemeinsam haben sie eine neue Leidenschaft entdeckt: Reisen mit dem eigenen Wohnmobil. Zwar nicht notwendigerweise, aber seiner Zielstrebigkeit entsprechend hat es Helmut Flachberger nicht gescheut, vor einem Jahr die Führerscheinprüfung für LKW und Anhänger abzulegen. Ein Professor darf es sich natürlich nicht leisten hier durchzufallen, weshalb er sich besonders intensiv darauf vorbereitet hat.

Der enge Kontakt zu den Studierenden und Absolventen zeichnet Prof. Flachberger aus und er kümmert sich ständig darum, diese zur Teilnahme an den Veranstaltungen des Bergmännischen Verbandes sowie zur Mitarbeit im Fachausschuss zu motivieren, um auch zukünftig einen umfangreichen Erfahrungsaustausch möglich zu machen.

Ich freue mich auf eine weiterhin gute Zusammenarbeit und Freundschaft.

Glück auf!

Günter Waldl

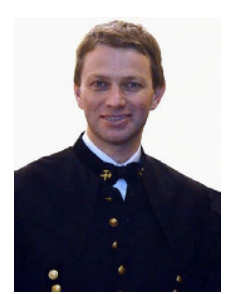

Dipl.-Ing. Dr. mont. Günter Waldl,

Leiter des Fachausschusses für Aufbereitung des BVÖ 